\title{
Eosinophil lysis and plasma extravasation
}

\section{To the Editor:}

I read with interest two recent Editorials, one by ALBERA and GHг [1] on eosinophil activation (in eosinophilic pneumonia) and the other by Kubes [2], which discusses plasma extravasation (in animal tissues). While these editorials no doubt deal extremely well with topical notions in these fields, there are a few additional aspects that might be of general interest.

With regard to eosinophil activation there is a potentially very important in vivo phenomenon that has been totally neglected so far [3]. I am referring to the eosinophil lysis process that quickly releases clusters of free eosinophil granules (Cfegs) [3]. Eosinophil lysis and generation of Cfegs are a feature of most eosinophilic diseases and can be induced acutely by inflammatory challenges in vivo [3]. The Cfegs, containing potent proteins, appear in disease tissues close to important target cells and organs [3]. Cfegs may also abound in the airway lumen [3]. Indeed, the common measurements of eosinophilic cationic protein (ECP) in cell-free samples of airway surface liquids may, to a high degree, reflect ECP contained in Cfegs [3]. The presence of Cfegs, that is the result of nonapoptotic death and disappearance of eosinophil cells, would also ruin simple correlations between analysed levels of ECP and eosinophil numbers in lumenal samples [3]. During the last $50 \mathrm{yrs}$, the occurrence of eosinophil lysis and Cfegs in eosinophilic pneumonia has been illustrated repeatedly [3]; one may even regard them as a typical feature of this disease [3]. Although Albera and GHio [1] are in the very best company, I wonder about the reasons for their failure to notice this phenomenon. Is it possible that Cfegs generation has been completely ignored by scientists around the world because it is an in vivo event that is outside the "all important" in vitro research paradigms?

I admire the ability of KuBEs [2] to deal succinctly with diverse data on tissue plasma changes reported to occur in response to nitric oxide active agents. However, with regard to airways plasma exudation responses there are several methodological and conceptual aspects that should, perhaps, be considered, particularly when findings from different laboratories are compared. For example, it may not be trivial whether workers have given drugs, mediators, etc., intravenously or topically, on the airway mucosa. ERJEFÄLT et al. [4], thus, found that topical L-NAME produced a potent exudative response that could not be mimicked by intravenous L-NAME. In the work that prompted the editorial by KuBES [2], only intravenous injections were employed [5].

Furthermore, it may be important to consider how socalled extravasation was actually analysed in this latter work [5]: 1) Only total tissue Evans blue dye was deter- mined [5]. This is a good measure of total albumin, but provides a less reliable distinction between the albumin that is extravascular and the albumin that inevitably remains intravascular. The claim that the intravascular pool in airway mucosa can be washed away [5], is not well founded, (see [6] and references cited therein); 2) Most importantly, the authors [2,5] fail to determine and discuss lumenal entry of plasma. This flaw is remarkable. Not only because lumenal entry of plasma is a major aspect of the original demonstration of airways plasma extravasation in response to L-NAME [4]. Neglecting the lumenal fate of extravasated plasma is unfortunate also in view of the fact that this is a quick and almost quantitative mode of elimination of the tissue plasma (briefly described below and most recently reviewed in [6]).

In human airways, as well as in guinea-pig trachea, lumenal entry of plasma proteins, ranging from albumin to $\alpha_{2}$-macroglobulin, starts within a minute after topical challenge of the mucosa [6]. The epithelial passage, through ubiquitous paracellular pathways, appears to be regulated by tiny increases in the hydrostatic pressure load (caused by the extravasated plasma itself) on the basolateral aspects of columnar epithelial cells [6]. There is an exceedingly elegant mechanism by which unsieved plasma can enter the airway lumen, without prior development of any oedema, harmful effects on the epithelial lining or compromising the function of the animal or human airway epithelial lining as an absorption barrier [6]. These findings identify the mucosal exudation response as, basically, a powerful first-line defence mechanism of the intact airway surface [6].

There is a potential link between the eosinophil aspect discussed above and the plasma exudation mechanisms. Eosinophil lysis and Cfegs generation evidently occurs in vivo and particularly at sites where the tissue is flooded with extravasated plasma [3]. I would not be surprised if, in the dynamic brew of multipotent plasma-derived proteins and peptides (a milieu not readily created in vitro), we will find molecular clues to the "ultimate activation" of eosinophils that characterize eosinophilic pneumonia, bronchial asthma and other eosinophilic diseases [3].

\section{References}

1. Albera C, Ghio P. Eosinophils in eosinophilic pneumonia. Eur Respir J 1997; 9: 2437-2439.

2. Kubes P. Nitric oxide and microvascular permeability: a continuing dilemma. Eur Respir J 1997; 10: 4-5.

3. Persson CGA, Erjefält JS. Eosinophil lysis and free granules: an in vivo paradigm for cell activation and drug development. TiPs 1997; 18: 117-123. 
4. Erjefält JS, Erjefält I, Sundler F, Persson CGA. Mucosal nitric oxide may tonically suppress airways plasma exudation. Am J Respir Crit Care Med 1994; 150: 227-232.

5. Kageyama N, Miura M, Ichinose M, et al. Role of endogenous nitric oxide in airway microvascular leakage induced by inflammatory mediators. Eur Respir J 1997; 10: $13-19$

6. Persson CGA, Erjefält JS, Andersson M, Greiff L,
Svensson C. Extravasation, lamina propria flooding and lumenal entry of bulk plasma exudate in mucosal defence, inflammation and repair. Pulm Pharmacol 1996; 9: $129-139$.

\section{C.G.A. Persson}

Dept Clinical Pharmacology, University Hospital of Lund, S-22185 Lund, Sweden.

\section{REPLY}

\section{From the authors:}

We thank Dr Persson for his comments on our editorial; in his letter Persson points out that in our paper there were no data on some aspects of eosinophil activation and degranulation.

The first consideration is that an editorial is not a review article and, consequently, does not consider the entire knowledge of the topic. In our paper we preferred to dedicate particular attention to the intricate functional relationships between other alveolar immunocompetent cells and eosinophils and to underline the heterogeneity of eosinophil recruitment and activation in idiopathic eosinophilic pneumonia (IEP) rather than focus our attention on the problem of mediators contained and released by eosinophil granules, which has been investigated in depth, as Persson himself affirms, in the last 50 yrs.

As far as the problem of clusters of free eosinophilic granules (Cfegs) generation is specifically concerned, the effects of eosinophilic inflammation in the lung are mainly mediated by the content of eosinophilic granules that are released near target tissues and organs by activated eosinophils in different ways, including degranulation of living cells and cell lysis in the necrotic, but not apoptotic pathway. Within target tissues, injury always occurs [1-3] where free extracelluar granules release eosinophilic cationic protein (ECP) and other mediators, such as tumour necrosis factor- $\alpha$ (TNF- $\alpha)$ [4] and cytokines [5], able to generate local damage, increase inflammation either in a paracrine and/or autocrine way, and induce alteration in local permeability to plasma components. So, tissue damage could develop, at least in part, independently from the way in which granules have been discharged by the cell, in all the tissues in which eosinophilic infiltration occurs.

In IEP patients, both lung damage and tissue flooding by extravased plasma could be generated by other mediators contained in eosinophil granules such as TNF- $\alpha$ [4], which is able to induce alterations of vascular permeability as well as tissue damage independently from ECP [6].

In addition, the ability of neuropeptides to activate human alveolar macrophages and to induce these cells to release superoxide anion [7] suggests that in IEP, neuropeptides, synthesized de novo and released after eosinophil activation, are probably able to mediate both lung parenchymal injury and the alteration of local permeability by their interaction with the alveolar population of these cells. The potential link between eosinophilic activation and plasma exudation mechanisms suggested by Persson seems to be confirmed by the presence of high levels of both albumin and D-dimer (respectively 40 and 10 fold higher than in healthy controls and 10 and twofold higher than in active sarcoidosis) found in bronchoalveolar lavage fluid from IEP patients (personal unpublished data, accepted for presentation at the Berlin European Respiratory Society congress).

These phenomena could further complicate the final picture of eosinophilic inflammation of the lung, and underline the relevance of both in vivo and in vitro studies for the complete understanding of eosinophil-mediated lung diseases.

\section{References}

1. Nonomura A, Kono N, Mizukami Y, Nakanuma Y. Histological changes of the liver in experimental graft versus host disease across minor histocompatibility barriers. VIII. Role of eosinophilic infiltration. Liver 1996; 16: $42-47$.

2. Chen KR, Pittelkow MR, Su D, Gleich J, Newman W, Leiferman KM. Recurrent cutaneous eosinophillic eosinophilic vasculitis: a novel eosinophil-mediated syndrome. Archiv Dermatol 1994; 130: 1159-1166.

3. Minnicozzi M, Duran WN, Gleich GJ, Egan RW. Eosinophil granule proteins increase microvascular micromolecular transport in the hamster cheek pouch. J Immunol 1994; 153: 2664-2670.

4. Beil WJ, Weller PF, Tzizik DM, Galli SJ, Dvorak AM. Ultrastructural immunogold localization of tumour necrosis factor-alpha to the matrix compartment of eosinophil secondary granules in patient with hypereosinophilic syndrome. J Histochem Cytochem 1993; 41: $1611-1615$.

5. Kroegel C, Virchow JC Jr, Luttmann W, Walker C, Warner JA. Pulmonary immune cells in health and disease: the eosinophil leucocyte (part I). Eur Respir J 1994; 7: 515-543.

6. Strieter RM, Kunkel SL, Bone RC. Role of tumour necrosis factor- $\alpha$ in disease states and inflammation. Crit Care Med 1993; 21: S447-S463.

7. Brunelleschi S, Guidotto S, Viano I, et al. Tachykinin activation of human alveolar macrophages in tobacco smoke and sarcoidosis: a phenotypical and functional study. Neuropeptides 1996; 30 (5): 456-464.

\section{Albera, P. Ghio}

University of Turin, Clinical and Biological Sciences Dept, San Luigi Hospital, Regione Gonzole 10, 10043 Orbassano, Turin, Italy. 